\title{
Post-intubation tracheal stenosis: monitoring is essential!
}

André Fidalgo 1, Telma Carlos 2, Eunice Silva 2, Elisabete Valente 3;

1- Anesthesiology resident; 2- Anesthesiology consultant; 3- Chief department Centro Hospitalar de Leiria, EPE

\section{Introduction}

Post-intubation tracheal stenosis (PITS) is an iatrogenic injury subsequent from the repair of a transmural injury of the trachea with cicatricial tissue formation and manifests as a fixed and severe thoracic restriction of pulmonary ventilation $^{(1,2)}$. Despite of the declining incidence on last decade $(1 \%)$ thanks to the introduction of new high-volume, low-pressure cuff endotracheal tubes, this continues to be a problem which impacts life quality, so prevention is fundamental $(3,4)$.

\section{Case Report}

\section{Personal History:}

- 59 year old woman;

- Allergies: seafood;

- Toxic habits: none;

- Pathological background: Crohn's D., Hypertension, thyroid nodule;

- Animal contact: rabbits, chickens, ducks, dogs, canary;

- Usual medication: Triplixam, Infliximab;

- Surgical history: 6 surgeries under GA with ET

\section{Medical History:}

Effort dyspnea and audible stridor, without wheezing, with one and half years of gradual evolution which was attributed to inhalation of sawdust powder. The patient didn't report fever, weight loss, anorexia, coughing or any kind of expectoration.

At physical examination she had no alterations on cardiac auscultation, although on pulmonary auscultation it was found an inspiratory stridor at the level of the superior airway.

ORL consultation: Direct laryngoscopy reported a subglottic tracheal stenosis.

\section{Diagnosis and Treatment (Pulmonology):}

Rigid bronchoscopy with identification of a subglottic complex stenosis $(1.5 \mathrm{~cm}$ inferior to vocal cords with $1 \mathrm{~cm}$ of caudal extension and 0.6 $\mathrm{cm}$ of luminal diameter). Dilatation and Vergnon prosthesis placement.

Combining medical history and the characteristics of the stenotic lesion they concluded the cause was related to the multiple endotracheal intubations performed.
The patient maintained recurrent complaints of dyspnea, odynophagia and purulent expectoration.

Another three bronchofibroscopies were necessary to evaluate the position and permeability of the prosthesis.

Due to persistence and complaint intolerance the patient underwent rigid bronchoscopy with prothesis removal.

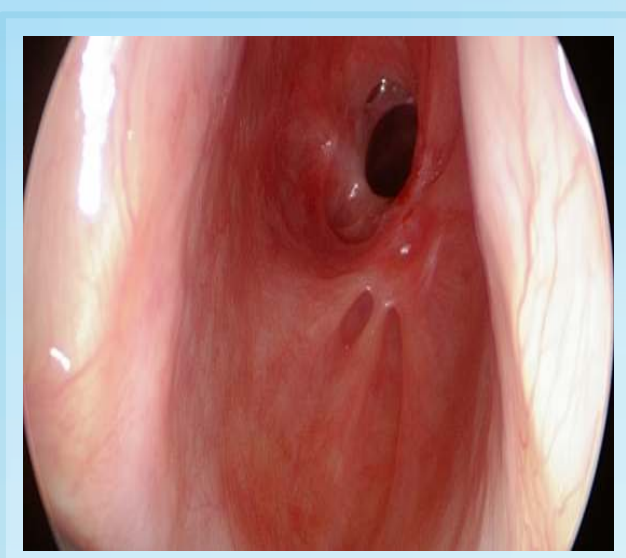

1 Subglottic stenosis;

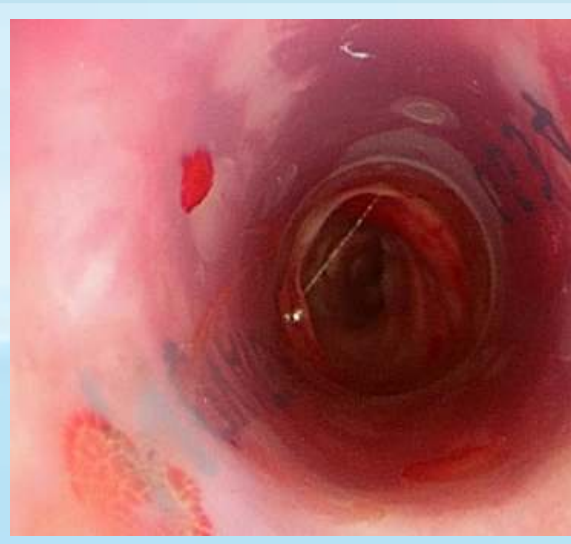

3. Intrastent;

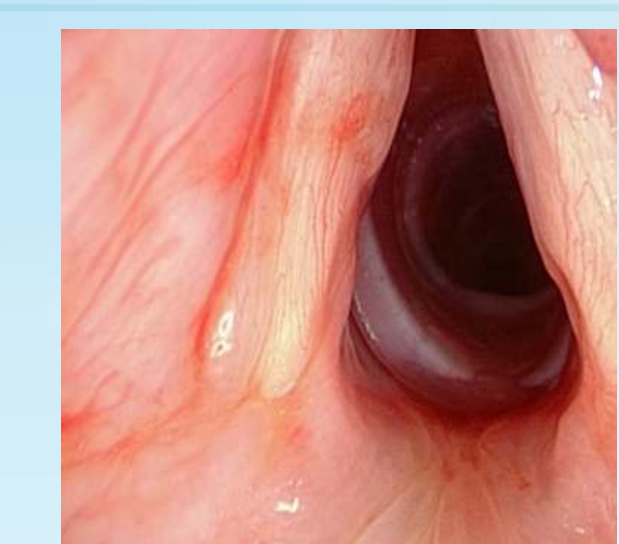

2. After stent placement

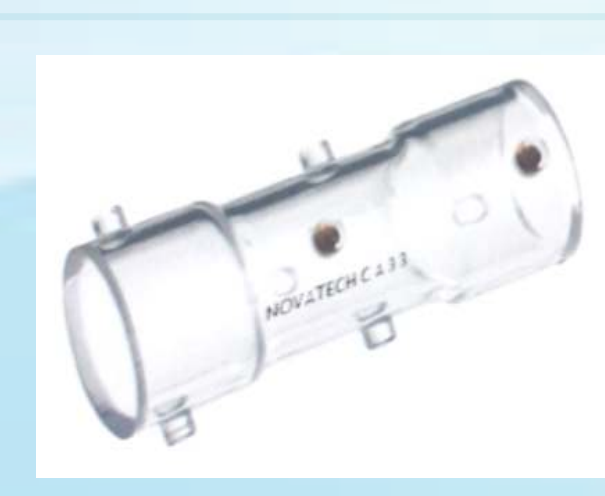

4. Vergnon prothesis Courtesy of Dr Salvato Feijó.

\section{Discussion}

Every patient proposed to surgery under GA with ET is susceptible to develop post-intubation tracheal stenosis which is the leading cause of tracheal stenosis(3). Potential associated risk factors are: female sex, obesity, diabetes, hypertension, ischemic heart disease, smoking multiple surgeries, prolonged ET placement steroid therapy, hypoperfusion and trauma resulting from ET placement ${ }^{(5)}$. The symptomatology can be misleading, making the physician diagnose it as asthma, whose conventional treatment only provides slight symptom relief(1,4). This case report shows some of the consequences of ET placement without cuff pressure control. The clinic and the high risk of relapse impose the need for multiple endoscopic interventions, thereby interfering greatly with the patient's life quality.

Risk factor assessment in patients undergoing GA with ET, cuff pressure monitoring $(<30 \mathrm{mmHg})$, and careful handling during extubation are crucial for prevention of tracheal stenosis.

References: 1. De, S., \& De, S. (2008). Post intubation tracheal stenosis. Indian Journal of Critical Care Medicine : Peer-Reviewed, Official Publication of Indian Society of Critical Care Medicine, 12(4), 194-197. http://doi.org/10.4103/0972-5229.45081; 2. Gelbard A, Francis DO, Sandulache VC, Simmons JC, Donovan DT, Ongkasuwan J. Causes and con consequent M, Chalouny G, Dabar G, Management or Postintubation Trach R. Aboul, B. Lo 233-237, ISSN 0422-7638, htp.//dx.dol.org/10.1016//.ejcd.2013.10.015; 5. ias, N., Choneou, A., Tabba, M. K., Gonzalez, A. V., Gray, A. W., Lamb, C. R., ... Beamis, J. F. (2008). Post tracheostomy and post intubation tracheal stenosis: Report of 31 cases and review of the literature. BMC Pulmonary Medicine, 8, 18. http://doi.org/10.1186/1471-2466-8-18 